\title{
Оценка кинетических и равновесных параметров сорбции сапонина природным сорбентом - хитозаном
}

\author{
Смусева С.О., Мироненко Н.В., Брежнева Т.А., Селеменев В.Ф. \\ ФГБОУ ВО «Воронежский государственный университет», Воронеж
}

Поступила в редакцию 26.03.2018 г.

DOI: https://doi.org/10.17308/sorpchrom.2018.18/502

Рассмотрена кинетика сорбции тритерпенового сапонина природным сорбентом хитозаном. Определена лимитирующая стадия сорбции - диффузия внутрь зерна сорбента. Рассчитаны коэффициенты внутренней диффузии и энергия активации процесса поглощения сапонина сорбентом. Представлены результаты исследования влияния температуры на сорбцию в равновесных условиях. Рассчитаны и обсуждены термодинамические параметры системы: свободная энергия Гиббса $(\Delta \mathrm{G})$, энтальпия $(\Delta \mathrm{H})$ и энтропия $(\Delta \mathrm{S})$.

Ключевые слова: сапонин, хитозан, сорбция, энергия активации, изотерма сорбции

\section{Estimation of kinetic and equilibrium parameters of sorption of saponin by a natural sorbent - chitosan}

\author{
Smuseva S.O., Mironenko N.V., Brezhneva T.A., Selemenev V.F. \\ Voronezh State University, Voronezh
}

Saponins have found application in practical medicine due to a wide range of biological and pharmacological activities. The studies of sorption of glycoside by synthetic and natural origin with sorbents have shown a complex multistage interaction mechanism, including ion-coordination, hydrogen and hydrophobic bonds. It is known that an increase in temperature leads to a change in the physicochemical properties of the sorbate and sorbent. For example, a change in the degree of dissociation of functional groups, solubility, displacement of the critical concentration of micelle formation, etc. On the other hand, the increase in temperature affects the properties of the sorbent - leading to an increase in swelling and enhance the protolysis of functional groups. In addition, as the temperature increases, the thermal motion of molecules is strengthened, the network of hydrogen bonds is loosened, which facilitates the weakening of interactions in the sorbent.

The aim of the work was to consider the thermodynamic and kinetic parameters of the sorption of the triterpene saponin Quillaja Saponaria Molina on the natural sorbent chitosan. The object of the study was a sample of Quillaja Saponaria Molina saponin from the USA. Chitosan is an aminopolysaccharide, a derivative of a linear polysaccharide whose macromolecule consists of bound $\beta-(1-4) \mathrm{D}$-glucosamine units and Nacetyl-D-glucosamine, is used as a biologically active food additive. Kinetic curves of saponin sorption from the solution were obtained using a limited volume method. Sorption equilibrium in the saponin-chitosan system was studied at a temperature of $276 \pm 2,298 \pm 2,353 \pm 2 \mathrm{~K}$ under static conditions using the variable concentration method.

The internal diffusion nature of the limitation is revealed, and the coefficients of internal diffusion are calculated. The resulting diffusion coefficients are values of the order of $10^{-13}-10^{-10} \mathrm{~cm}^{2} / \mathrm{s}-$ lower than in polymeric sorbents, which is due to the stiffness of the matrix and the pore diameter. Depending on the concentration of saponin in the solution, the kinetics of the sorption process by chitosan will be hydrationdehydration processes at low concentrations and micellization at large.

The activation energy of sorption $\mathrm{E}_{\mathrm{akt}}=76 \mathrm{~kJ} / \mathrm{mol}$ is calculated. This value confirms the determining contribution of internal diffusion to the general nature of the limiting stage when triterpene saponin interacts 
with a natural sorbent with chitosan. The calculation of the thermodynamic parameters showed that an increase in temperature leads to a decrease in the adsorption equilibrium constant, which indicates a decrease in the energy of interaction of the saponin molecules with the surface of the sorbent. The values of the interaction energy between saponin and chitosan are in the range $-\Delta G=18.1 \div 19.4 \mathrm{~kJ} / \mathrm{mol}$, which is typical for physical adsorption. The process of saponin absorption is exothermic, which is typical for most systems organic sorbent - sorbent. Positive changes in entropy during sorption on chitosan are related to the hydrophobic effect.

Keywords: saponin, chitosan, sorption activation energy, sorption isotherms

\section{Введение}

Сапонины нашли применение в практической медицине благодаря широкому спектру биологической и фармакологической активности. Проведенные исследования сорбции гликозида сорбентами синтетического и природного происхождения показали сложный многоступенчатый механизм взаимодействия, включающий ионно-координационные, водородные и гидрофобные связи [1]. С помощью компьютерного моделирования было показано, что определяющую роль в вовлечении гликозида в спиральную конформацию хитозана играют водородные связи, значительно превышающие по силе ионное взаимодействие. Аналогичные результаты получены при работе с другими высокомолекулярными соединениями белковой природы [2]. Поверхностная активность сапонина, проявляющаяся в возможности образования в фазе раствора и сорбента ассоциатов - мицелл, свидетельствует о влиянии на ход сорбционного процесса образования гидрофобных связей, роль которых, исходя из литературных данных [3], на сегодняшний день до конца не выяснена. Известно, что повышение температуры приводит к изменению физикохимических свойств сорбата и сорбента. Например, изменению степени диссоциации функциональных групп, растворимости, смещению области критической концентрации мицеллообразования и др. С другой стороны, повышение температуры влияет на свойства сорбента - приводит к увеличению набухания и усилению протолиза функциональных групп [4]. Кроме того, необходимо учитывать, что при повышении температуры усиливается тепловое движение молекул, разрыхляется сетка водородных связей, что способствует ослаблению взаимодействий в сорбенте [5]. Таким образом, рассмотрение термодинамических и кинетических параметров сорбции позволит внести ясность в физико-химическую природу протекания сорбционного процесса «сапонин-хитозан».

\section{Эксперимент}

Объектом исследования являлся образец сапонина Quillaja Saponaria Molina производства США, представляющий собой желтый аморфный порошок, обладающий горьким, острым вкусом. Характерное свойство сапонинов - поверхностная активность [6], выражающаяся в способности давать легко пенящиеся коллоидные растворы. Анализ образца методом ТСХ в системе «н-бутанол - вода - уксусная кислота» (4:5:1) показал наличие одной зоны индивидуального гликозида, структура которого приведена на рис. 1. Основные физико-химические характеристики образца гликозида по данным [7-8] приведены в таблице 1.

В качестве сорбента выступал хитозан, используемый как биологически активная добавка к пище, производства ООО «Фармакон Продакшн» (СанктПетербург). Средняя молекулярная масса 250-300 кДа, степень дезацетилирования $89.6 \%$, размер зерна 10-30 мкм. Структурная формула приведена на рис. 2.

Кинетические кривые сорбции сапонина из раствора получали, используя метод ограниченного объема [9]. Навески воздушно-сухого хитозана массой $1.0 \pm 0.0002$ 
г заливали в конической колбе с притертой крышкой $1000 \mathrm{~cm}^{3}$ раствора сапонина с концентрациями 0.009-0.87 ммоль/дм³. На анализ отбирали раствор через определенные промежутки времени от момента начала опыта. Отобранные аликвоты анализировали на содержание сапонина методом УФ-спектрофотометрии [10-11].

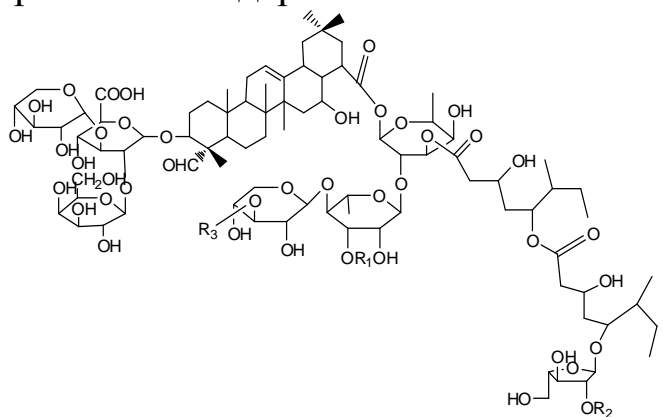

Рис. 1. Общая структурная формула сапонинов Quillaja Saponaria Molina $\left(\mathrm{R}_{1}-\mathrm{C}_{9} \mathrm{H}_{12} \mathrm{O}_{9}, \mathrm{R}_{2}-\mathrm{C}_{6} \mathrm{H}_{12} \mathrm{O}_{5}, \mathrm{R}_{3}-\mathrm{C}_{5} \mathrm{H}_{10} \mathrm{O}_{5}\right.$ (апиоза))

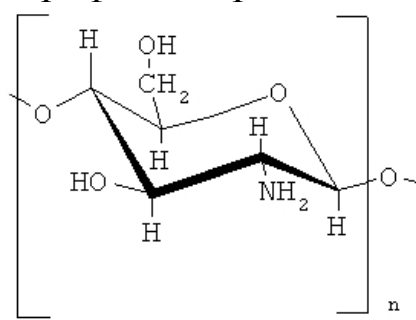

Рис. 2. Структурная формула звена хитозана

Таблица 1. Характеристики тритерпенового сапонина Quillaja Saponaria Molina

\begin{tabular}{|c|c|}
\hline Молекулярная масса, г/моль & 2321 \\
\hline $\mathrm{pH}$ & 4.5 \\
\hline $\mathrm{pK}$ (глюкуроновая кислота) & 3.18 \\
\hline Растворимость, мг $/ \mathrm{cm}^{3} \mathrm{H}_{2} \mathrm{O}$ & 40 \\
\hline Критическая концентрация мицеллообразования: ККМ, ммоль/дм ${ }^{3}$ & $0.26-0.35$ \\
\hline
\end{tabular}

Сорбционное равновесие в системе сапонин - хитозан изучали при температурах $276 \pm 2,298 \pm 2,353 \pm 2 К$ в статических условиях методом переменных концентраций. Навески сорбента массой $0.2 \pm 0.0002$ г в воздушно-сухом состоянии заливали в конических колбах с притертой крышкой растворами сапонина объемом 200 мл с концентрациями 0.01-17.00 ммоль/дм³ . Содержимое колб выдерживали при перемешивании в течение 4 часов до установления равновесия в системе. Отобранные аликвоты анализировали на содержание сапонина спектрофотометрически.

\section{Обсуждение результатов}

В [12] установлено, что ход кинетических кривых сорбции сапонина на хитозане зависит от концентрации гликозида и определяется структурными перегруппировками сорбата в фазе сорбента и раствора. Особый интерес вызывает рассмотрение сорбции сапонина хитозаном до и после образования мицелл (ККМ) в растворе с целью изучения влияния мицеллярной фазы на скорость и механизм сорбции, учет которой при описании вклада диффузии крайне затруднителен. Очевидно, что после ККМ затрудняется движение сапонина вглубь зерна из-за способности его образовывать в растворе мицеллы, препятствующие диффузии молекул в поры сорбента.

Сорбционный процесс имеет сложный и многостадийный характер, и рассмотрение всех стадий этого процесса вкупе трудноосуществимо. Поэтому, обычно, прибегают к ряду упрощений, используя принцип лимитирующей стадии. В качестве лимитирующей стадии рассматривают либо внешнюю, либо внутреннюю диффузию. Графические способы, используемые в качестве критерия оценки вклада каждой из типов диффузий в большинстве литературных источников данных [13-14], основаны на решении уравнения диффузии в шар с граничным условием I рода:

$$
\mathrm{M}_{\mathrm{t}}=2 \mathrm{~A}\left(\mathrm{C}_{\infty}-\mathrm{C}_{0}\right) \sqrt{\mathrm{D}} \mathrm{t} / \mathrm{n} \text {, }
$$


где $\mathrm{A}$ - площадь участка поверхности тела, $\mathrm{C}_{\infty}$ - концентрация в момент наступления равновесия, $\mathrm{C}_{0}$ - исходная концентрация вещества, D - коэффициент диффузии, $\mathrm{t}$ - время. Из уравнения (1) следует, что получаемая линейная зависимость $\mathrm{F}$ от $\sqrt{\mathrm{t}}$ при малых значениях времени является достоверным признаком внутренней диффузии.

На рис. 3 представлены линейные зависимости F от $\mathrm{t}$ с соответствующими коэффициентами корреляции.

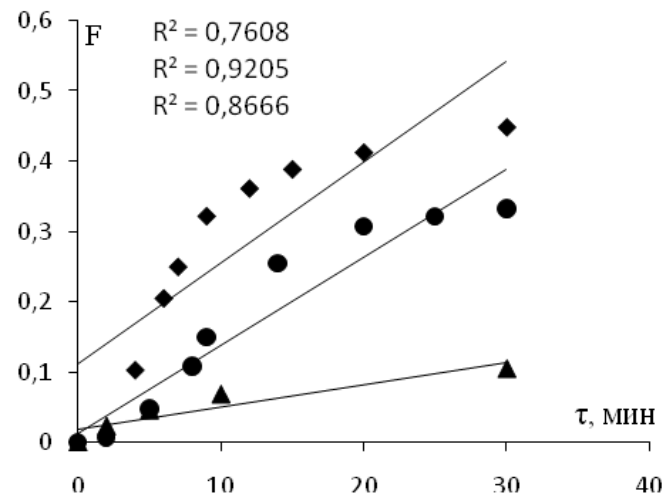

Рис. 3. Зависимость F от t, $1-\mathrm{c}=9.5 \cdot 10^{-3}$ ммоль $/$ дм $^{3}, 2-\mathrm{c}=2.4 \cdot 10^{-2}$ ммоль $/$ дм $^{3}$, $3-\mathrm{c}=4.7 \cdot 10^{-2}$ ммоль/дм ${ }^{3}$.

Выход на прямую может означать переход процесса от неупорядоченного режима, в котором распределение концентрации существенно определяется начальным распределением вещества к регулярному режиму, определяемому лишь размерами зерен и его диффузионными параметрами [13]. Таким образом, можно говорить о внутридиффузионном механизме поглощения сапонина хитозаном, что вполне закономерно.

В классическом варианте для расчета коэффициентов внутренней диффузии используют уравнение Патерсона в модификации Бойда [15]:

$$
F_{i}=1-\frac{6}{\pi^{2}} \sum \frac{1}{n^{2}} \exp \left(-\pi^{2} n F o_{i}\right),
$$

где, $F_{i}$ - степень заполнения сорбционной емкости, $F o_{i}-$ безразмерное время или критерий Фурье $\left(F o_{i}=\frac{\bar{D} \tau_{i}}{r^{2}}\right), \tau_{i}-$ время достижения величины $F_{i}, \mathrm{r}-$ радиус гранул сорбента, $\bar{D}$ - коэффициент внутренней диффузии.

На практике гораздо чаще используют простое апроксимационное выражение согласно [16], которое с высокой точностью соответствует данным уравнения (3):

$$
\mathrm{Bt}_{\mathrm{i}}=-2 \mathrm{~F}_{\mathrm{i}} \lg \left(1-\mathrm{F}_{\mathrm{i}}\right), \mathrm{B}=\pi^{2} \bar{D} / \mathrm{r}^{2} \text {, }
$$

где $\mathrm{t}$ - время, $\mathrm{r}$ - радиус частицы. Уравнения $(4,5)$ наиболее часто используются для расчета коэффициента диффузии для каждого значения $\mathrm{F}$ с высокой точностью при $\mathrm{F}=0.05-0.95$, ошибка усреднения по всей кривой при этом не превышает 10-18\%:

$$
\bar{D}=\frac{-2 r^{2} F \lg (1-F)}{\pi^{2} t}
$$

Получаемые коэффициенты диффузии представляют собой величины порядка $10^{-13}-10^{-10} \mathrm{~cm}^{2} /$ сек. Рассчитанные таким способом значения коэффициента диффузии приведены в табл. 2.

Порядок получаемых величин составляет $10^{-13}-10^{-10}$ - ниже, чем на полимерных сорбентах, что обусловлено жесткостью матрицы и диаметром пор [17-18].

Возрастание значений коэффициента диффузии с увеличением концентрации, вероятно, обусловлено сжатием сорбента вследствие его дегидратации, показанной нами ранее в работе [12]. Можно полагать, что, вследствие периодических процессов сжатия-набухания хитозана в ходе сорбции сапонина, разброс величин коэффициен- 
тов диффузии будет достаточно широким и может только качественно характеризовать кинетику процесса в данной системе.

Таблица 2. Величины коэффициентов внутренней диффузии

\begin{tabular}{|c|c|c|}
\hline \multicolumn{2}{|c|}{ Концентрация сапонина, ммоль/дм ${ }^{3}$} & $\mathrm{D}, \mathrm{cm}^{2} / \mathrm{ce \kappa}^{-13}$ \\
\hline \multirow{3}{*}{ До ККМ } & $0.9 \cdot 10^{-3}$ & $5.79 \cdot 10^{-13}$ \\
\cline { 2 - 3 } & $2.4 \cdot 10^{-2}$ & $4.61 \cdot 10^{-12}$ \\
\cline { 2 - 3 } & $4.7 \cdot 10^{-2}$ & $1.48 \cdot 10^{-11}$ \\
\hline \multirow{2}{*}{ После ККМ } & $4.8 \cdot 10^{-1}$ & $1.16 \cdot 10^{-10}$ \\
\cline { 2 - 3 } & $9.5 \cdot 10^{-1}$ & $1.20 \cdot 10^{-10}$ \\
\hline
\end{tabular}

Поскольку в адсорбционном слое при С<ККМ присутствуют как молекулы ПАВ, так и молекулы воды, то изменение концентрации в объеме раствора аналогично изменению соотношения компонентов раствора в адсорбционной фазе. На этом основании можно полагать, что ассоциация в адсорбционном слое ПАВ наступает при достижении некоторой концентрации еще до того, как в объеме растворе будет достигнута ККМ [19-20]. Большие органические ионы при диффузии вглубь зерна частично раздвигают подвижные звенья набухшего сорбента и вытесняют часть воды, находящейся в нем, преодолевают взаимодействие с матрицей и функциональными группами сорбента. После равновесной концентрации ККМ процессы структуризации и упорядочивания в фазе хитозана слабо влияют на гидратационные характеристики сорбента, так что процесс дегидратации и процесс удерживания воды в фазе сорбента уравновешивают друг друга. Поэтому незначительное изменение коэффициента диффузии после ККМ может быть связано со снижением проницаемости зерна сорбента по мере заполнения его сапонином из-за влияния стерического фактора (размера мицелл), а также затруднением диффузии вглубь хитозана вследствие его набухания. Таким образом, в зависимости от концентрации сапонина в растворе, определяющими кинетику процесса сорбции хитозаном будут процессы гидратации-дегидратации при малых концентрациях и мицеллообразования - при больших.

Одним из факторов, влияющим на сорбцию ПАВ является температура, при которой протекает процесс. Важной характеристикой процесса сорбции является энергия активации, позволяющая оценить и обосновать вклад сил межмолекулярного взаимодействия в общий процесс поглощения вещества. Для ее расчета данные эксперимента представлялись в виде графической зависимости, описываемой уравнением:

$$
F=B+Z \cdot \tau,
$$

где Z - кажущаяся константа скорости процесса сорбции, В - свободный член уравнения, соответствующий участку, отсекаемому на оси ординат.

Кажущиеся константы при различных температурах определялись как тангенс угла наклона полученных зависимостей.

Затем, зная эти величины, рассчитывали энергию активации сорбции по уравнению Аррениуса [21]:

$$
k=A e^{\frac{-E_{a}}{R T}},
$$

представляя уравнение в логарифмической форме:

$$
\lg k=\lg B-\frac{E}{2,3 R} \frac{1}{T}
$$

График зависимости константы скорости от температуры в координатах $\lg k-$ $1 / T$ представляет собой прямую линию, отсекающую на оси ординат отрезок, равный $\lg B$, а тангенс угла наклона прямой позволяет определить энергию активации: $E_{\text {акт }}=-$

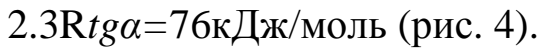

Смусева и др. / Сорбционные и хроматографические процессы. 2018. Т. 18. № 2 


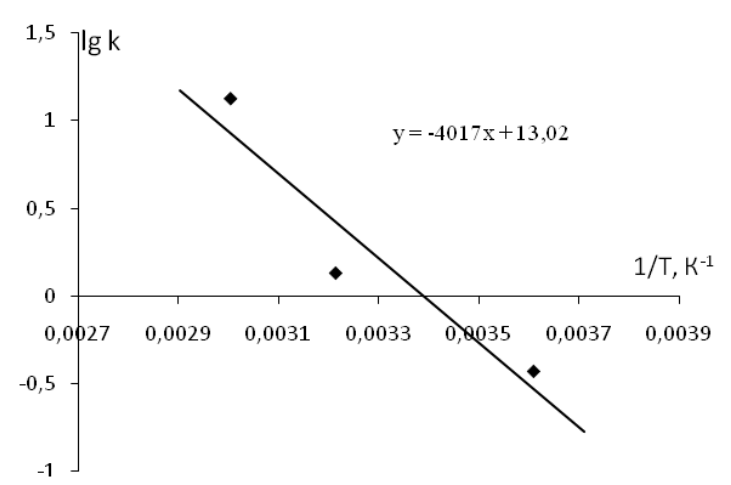

Рис. 4. Зависимость константы скорости от температуры

Полученное значение энергии активации подтверждает определяющий вклад внутренней диффузии в общий характер лимитирующей стадии при взаимодействии тритерпенового сапонина с природным сорбентом хитозаном. Представляет интерес изучение энергетических характеристик сорбции, условий ее протекания, а также возможности оптимизации процесса в зависимости от термодинамических параметров системы: свободной энергии Гиббса $(\Delta \mathrm{G})$, энтальпии $(\Delta \mathrm{H})$ и энтропии $(\Delta \mathrm{S})$.

На рис. 5 представлены изотермы сорбции сапонина Quillaja Saponaria Molina на хитозане при 298, 313 и 353 К. Полученные результаты свидетельствуют о влиянии температуры на равновесные характеристики сорбции. Из рис. 5. видно, что вид изотермы сорбции меняется незначительно, что свидетельствует о сохранении в целом механизма поглощения в разных температурных условиях.

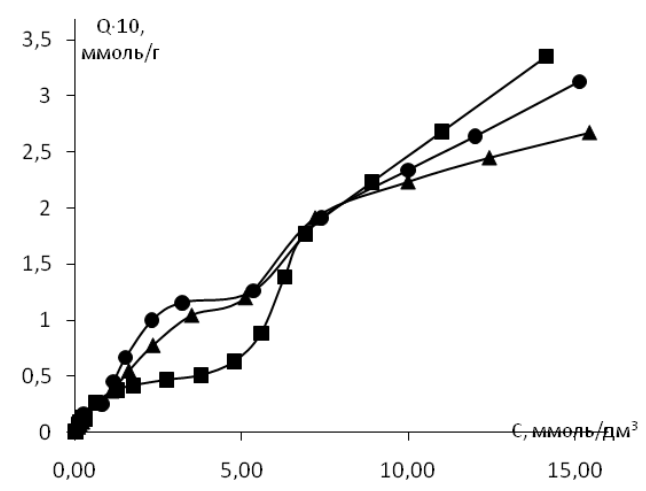

Рис. 5. Изотермы сорбции сапонина на хитозане при температурах $1-298 \mathrm{~K}, 2-313 \mathrm{~K}, 3-353 \mathrm{~K}$.

По экспериментальным значениям коэффициента сорбционного равновесия оценивали термодинамические функции сорбции. Так, значения коэффициента сорбционного равновесия $\left(\mathrm{K}_{\mathrm{L}}\right)$ полученные при решении уравнения типа БЭТ графическим способом позволили рассчитать энтальпию сорбции сапонина $(\Delta \mathrm{H})$ хитозаном по уравнению Вант-Гоффа:

$$
\Delta H=\frac{R T_{1} T_{2}}{T_{2}-T_{1}} \ln \left(\frac{K_{L 2}}{K_{L 1}}\right)
$$

где $\mathrm{K}_{\mathrm{L} 1}$ и $\mathrm{K}_{\mathrm{L} 2}$, - коэффициенты сорбционного равновесия при температуре системы $\mathrm{T}_{1}$ и $\mathrm{T}_{2}$ соответственно, $\mathrm{K} ; \mathrm{R}=8,31$ Дж/(моль $\left.\cdot \mathrm{K}\right)$ - универсальная газовая постоянная.

Следует отметить, что величина $\mathrm{K}_{\mathrm{L}}$ является формальной величиной, так как зависит от способа выражения концентрации [22]. Экспериментальный коэффициент сорбционного равновесия имеет размерность. Его подстановка в термодинамическое соотношение: 


$$
\Delta G=-R T \ln K^{\prime}
$$

означает, что равновесные концентрации нормированы относительно стандартного состояния $C_{p}{ }^{\prime}=C_{p} / a_{p}^{0}$, когда $a_{p}^{0}=1$ моль $/$ дм $^{3}$, в результате преобразования $K_{s} \rightarrow K$ '.

Величину энтропийного вклада $(\Delta S)$ сорбции рассчитывали по уравнению:

$$
\Delta S=\frac{\Delta H-\Delta G}{T}
$$
таблице 3.

Полученные равновесные характеристики сорбции сапонина представлены в

Таблица 3. Равновесные характеристики сорбции в системе «водный раствор сапонина - хитозан»

\begin{tabular}{|c|c|c|c|c|}
\hline $\mathrm{T}, \mathrm{K}$ & $\mathrm{K}^{\prime}$ & $\Delta \mathrm{H}, \mathrm{\kappa Д} /$ моль & $\Delta \mathrm{G}, \mathrm{\kappa Д} /$ моль & $\mathrm{T} \cdot \Delta \mathrm{S}$, кДж/моль \\
\hline 298 & 1520 & \multirow{2}{*}{-14.9} & -18.1 & 3.2 \\
\cline { 1 - 2 } 313 & 1140 & \multirow{2}{*}{-11.4} & -18.3 & 3.4 \\
\cline { 2 - 3 } & 740 & & -19.4 & 8.0 \\
\hline
\end{tabular}

Расчёты показали (табл. 3), что повышение температуры приводит к уменьшению константы адсорбционного равновесия, что свидетельствует о снижении энергии взаимодействия молекул сапонина с поверхностью сорбента. Отрицательные значения энергии Гиббса $(\Delta \mathrm{G})$ свидетельствуют о самопроизвольном протекании процесса сорбции. Значения энергии взаимодействия между сапонином и хитозаном находятся в интервале $-\Delta G=18.1 \div 19.4$ кДж/моль, что характерно для физической адсорбции (10-40 кДж/моль) [23]. Процесс поглощения сапонина является экзотермическим, что характерно для большинства систем органический сорбтив - сорбент.

Положительные изменения энтропии при сорбции на хитозане связаны с гидрофобным эффектом: образованием упорядоченных ансамблей из молекул воды вокруг углеводородных цепей в растворе и их разрушением при выходе углеводородного радикала на межфазную поверхность. Выход из воды молекул ПАВ в адсорбционный слой сопровождается ростом энтропии системы за счет разрушения «айсберговых» структур вокруг углеродных цепей молекул сапонина [3].

\section{Заключение}

Таким образом, установлено, что при сорбции сапонина Quillaja Saponaria Molina хитозаном лимитирующей стадией процесса является внутренняя диффузия; с ростом концентрации внешнего раствора увеличиваются коэффициенты внутренней диффузии сорбируемого вещества. Рассчитанное значение энергии активации подтверждает определяющий вклад внутренней диффузии в общий характер лимитирующей стадии.

Показано, что повышение температуры приводит к снижению энергии взаимодействия молекул сапонина с поверхностью сорбента. Установлено, что процесс поглощения сапонина экзотермический; положительные изменения энтропии при сорбции на хитозане связаны с гидрофобным эффектом.

\section{Список литературы}

1. Мироненко Н.В., Смусева С.О., Брежнева Т.А., Селеменев В.Ф и др. // Коллоидный журнал. 2017. Т. 79. № 2. С. 166-173.
2. Ламри Р. Структура и стабильность биологических макромолекул. Пер. с англ. М.В. Волькенштейна. М. Мир. 1973. 584 с. 
3. Измайлова В.Н., Ребиндер П.А. Структурообразование в белковых системах. М. Наука. 1974. 268 с.

4. Загородний А.А., Хохлов В.Ю., Селеменев В.Ф. и др. // Журнал физической химии. 1995. Т. 69. № 4. С. 661-663.

5. Эрдей-Груз Т. Явления переноса в водных системах. М. Мир. 1976. 594 с.

6. Куркин В.А. Фармакогнозия: Учебник для студентов фармацевтических вузов. Самара. Офорт. 2007.528 с.

7. Мироненко Н.В., Брежнева Т.А., Пояркова Т.Н., Селеменев В.Ф. // Хим.-фарм. журн. 2010. Т. 44. № 3. С. 48.

8. Mitra S., Dunga S.R. // Journal Agricultuzal and Food Chemistry. 1997. № 45. P. 1587.

9. Селеменев В.Ф., Славинская Г.В., Хохлов В.Ю., Чикин Г.А. Практикум по ионному обмену. Воронеж. Изд-во Воронеж. ун-та. 1999. $173 \mathrm{c}$.

10.Мироненко Н.В., Брежнева Т.А., Селеменев В.Ф. // Химия растительного сырья. 2011. № 3. C. 153-157.

11.Мироненко Н. В., Брежнева Т. А., Михина И. А., Селеменев В. Ф. // Заводская лаборатория. Диагностика материалов. 2009. Том 75. № 5. С. 19-23.

12.Мироненко Н.В., Брежнева Т.А., Шкутина И.В., Селеменев В.Ф. // Сорбционные и хроматографические проиессы. 2015. Т. 15. № 1. C. 74.

\section{References}

1. Mironenko N.V., Smuseva S.O., Brezhneva T.A., Selemenev V.F et al., Kolloidnyj zhurnal, 2017, Vol. 79, No 2, pp. 166-173.

2. Lamri R. Struktura i stabil'nost' biologicheskih makromolekul. Per. s angl. M.V. Vol'kenshtejna, M., Mir, 1973, 584 p.

3. Izmajlova V.N., Rebinder P.A. Strukturoobrazovanie $\mathrm{v}$ belkovyh sistemah, M., Nauka, 1974, $268 \mathrm{p}$.

4. Zagorodnij A.A., Khohlov V.YU., Selemenev V.F. e al., Zhurnal fizicheskoy khimii, 1995, Vol. 69, No 4, pp. 661-663.

5. Erdej-Gruz T., Yavleniya perenosa v vodnyh sistemah, M., Mir, 1976, 594 p.

6. Kurkin V.A. Farmakognozija: Uchebnik dlja studentov farmacevticheskih vuzov, Samara, Ofort, 2007, 528 p.
13.Кокотов Ю.А., Пасечник В.А. Равновесие и кинетика ионного обмена. Л. Химия. Ленингр. отд-ние. 1970. 336 с.

14.Кокотов Ю.А., Золотарев П.П., Елькин Г.Э. Теоретические основы ионного обмена. Сложные ионообменные системы. Л. Химия Ленинградское отд. 1986. 280 с.

15.Лейкин Ю.А, Тарасов Т.И., Тихонова Л.А., Муса Н. // Журнал Физической химии.1997. Т.71. №2. С.321.

16.Знаменский Ю.П. // Журнал физической химии.1993.Т.67. №9. С. 1924.

17.Мироненко Н.В., Михина И.А., Брежнева Т.А., Селеменев В.Ф. // Сорбиионные и хроматографические прочессы. 2008. Т.8. Вып.3. С.513.

18.Мироненко Н.В., Брежнева Т.А., Селеменев В.Ф. // Журнал физической химии. 2013. T. 87. № 3. С. 387.

19.Когановский А.М. Клименко Н.А. Адсорбция органических веществ из воды. Л. Химия, 1990. 256 с.

20.Хохлов А.Р, Дормидонтова Е.Е. // Уcneхи физических наук. 1997. Т.167. № 2. С. 113128.

21.Герасимов Я.И., Древинг В.П., Еремин Е.Н. Курс физической химии. Т. 2. М. Наука. 1970. $592 \mathrm{c}$.

22.Воронюк И.В., Елисеева Т.В., Текучев А.Ю. // Сорбиионные и хроматографические nроиессы. 2010. Т. 10. № 4. С. 618-622.

23.Котова Д.Л., Васильева С.Ю., Крысанова Т.А. // Журнал физической химии. 2014. Т. 88. № 8. C. 1223-1227.

7. Mironenko N.V., Brezhneva T.A., Poyarkova T.N., Selemenev V.F., Khim.-farm. zhurn., 2010, Vol. 44, No 3, pp. 48.

8. Mitra S., Dunga S.R., Journal Agricultuzal and Food Chemistry, 1997, No 45, pp. 1587.

9. Selemenev V.F., Slavinskaya G.V., Khokhlov V.Yu., Chikin G.A., Praktikum po ionnomu obmenu, Voronezh, Izd-voVoronezh. un-ta, 1999, $173 \mathrm{p}$.

10. Mironenko N.V., Brezhneva T.A., Selemenev V.F., Himija rastitel'nogo syr'ja, 2011, No 3, pp. 153-157.

11.Mironenko N.V., Brezhneva T.A., Mihina I.A., Selemenev V.F., Zavodckaja labopatopija. Diagnoctika matepialov, 2009, Vol. 75, No 5, pp. 19-23.

12.Mironenko N.V., Brezhneva T.A., Shkutina I.V., Selemenev V.F., Sorbtsionnye i khro- 
matograficheskie protsessy. 2015. Vol. 15, No 1, pp. 74-84.

13.Kokotov Ju.A., Pasechnik V.A. Ravnovesie i kinetika ionnogo obmena, L., Himija, Leningr. otd-nie, 1970, $336 \mathrm{p}$.

14.Kokotov Ju.A., Zolotarev P.P., El'kin G.Je.

Teoreticheskie osnovy ionnogo obmena. Slozhnye ionoobmennye sistemy, L., Himija Leningradskoe otd., 1986, $280 \mathrm{p}$.

15.Lejkin Ju.A, Tarasov T.I., Tihonova L.A., Musa N., Zhurnal Fizicheskoj himii, 1997, Vol. 71, No 2, $321 \mathrm{p}$.

16.Znamenskij Ju.P., Zhurnal fizicheskoj himii, 1993, Vol. 67, No 9, pp. 1924.

17.Mironenko N.V., Mihina I.A., Brezhneva T.A., Selemenev V.F., Sorbtsionnye i khromatograficheskie protsessy, 2008, Vol8, No 3, pp. 513.

18. Mironenko N.V., Brezhneva T.A., Selemenev V.F., Zhurnal fizicheskoi khimii, 2013, Vol. 87, No 3, pp. 387-390.

Смусева Светлана Олеговна - студентка химического факультета Воронежского государственного университета, Воронеж

Мироненко Наталья Владимировна к.Х.н., ассистент кафедры аналитической химии Воронежского государственного университета, Воронеж

Брежнева Татьяна Александровна к.фарм.н., доцент кафедры фармхимии и фармтехнологии Воронежского государственного университета, Воронеж

Селеменев Владимир Федорович - д.Х.н., проф., зав. каф. аналитической химии химического факультета Воронежского государственного университета, Воронеж, т.(473)2208-932
19.Koganovskii A.M., Levchenko T.M., Kirichenko V.A., Adsorbtsiya rastvorennykh veshchestv, Kiev, Naukova dumka, 1977, 223 p. 20.Hohlov A.R, Dormidontova E.E., Uspehi fizicheskih nauk, 1997, Vol. 167, No 2, pp. 113128.

21.Gerasimov Ja.I., Dreving V.P., Eremin E.N., Kurs fizicheskoj himii, Vol. 2, M., Nauka, 1970, $592 \mathrm{p}$.

22.Voronyuk I.V., Eliseeva T.V., Tekuchev A.Yu., Sorbtsionnye $i$ khromatograficheskie protsessy, 2010, Vol. 10, No 4, pp. 618-622.

23. Kotova D.L., Vasil'eva S.Ju., Krysanova T.A., Zhurnal fizicheskoj himii, 2014, Vol. 88, No 8, pp. 1223-1227.

24.Izmajlova V.N., Rebinder P.A., Strukturoobrazovanie v belkovyh sistemah, M., Nauka, 1974, $268 \mathrm{p}$.

Smuseva Svetlana O. - the student of chemical faculty, Voronezh State University, Voronezh, e-mail: svetlana_smuseva@mail.ru

Mironenko Natalia V. - the assistant of the department of Analitical chemistry, Voronezh State University, Voronezh, e-mail: natashamir@yandex.ru

Brezhneva Tatiana A. - the senior lecture of department of pharmaceutical chemistry and pharmaceutical technology, Voronezh State University, Voronezh

Selemenev Vladimir F. - the professor, head of the department of Analitical chemistry, Voronezh State University, Voronezh 